\title{
Research on Ebola Virus Prorogation Prediction based on Polymorphic Ant Colony Algorithm
}

\author{
Benfa Zou \\ College of Information Science and Engineering \\ Shandong University of Science and Technology \\ Qingdao, China, 266510
}

\author{
Benyou Zou \\ SINOPEC Research Institute of Petroleum \\ Engineering \\ Beijing, China, 100101
}

\begin{abstract}
The Ebola virus is a highly infectious and rare virus. In order to control the further spread of the Ebola virus, make the vaccine efficiently and quickly transport to the hardest hit by the disease, reduce constantly the number and scale of the Ebola epidemic, and ultimately control the spread of the virus, we use the shortest path to solve this problem. First, the model of shortest path problem is established. Then the polymorphic ant colony algorithm is applied to accomplish shortest path problem. Finally, it is showed in the simulation experiments that the PACA can reasonable to solve shortest path problem in Ebola virus prorogation, and it has a great advantage in search time, convergence speed, and optimization result and algorithm efficiency.
\end{abstract}

Keywords: Ebola Virus; Shortest Path; ant colony algorithm; polymorphic ant colony algorithm

\section{INTRODUCTION}

The Ebola virus is a highly infectious and rare virus [1]. Discovered its existence in southern Sudan and the Congo (DRC) (formerly Zaire) the Ebola River in 1976 which caused widespread concern and attention of the medical field, the name of "Ebola" is created. Ebola is a virus of the high mortality rate that is up to $90 \%$.

2014 Ebola outbreak [2] in West Africa is a large-scale virus outbreaks began in February. As of December 2, 2014, World Health Organization declared Guinea, Liberia, Sierra Leone, Mali the United States as well as Nigeria, Senegal and Spain that the epidemic has ended had appeared cumulative confirmed, probable, and possible Ebola infections 17,290 cases, of which 6128 people died. As of December 17, 2014, the World Health Organization (WHO) published data that the cases of Ebola infection (including suspected cases) of Liberia, Sierra Leone and Guinea in West Africa Ebola has reached 19,031 people, including the dead toll reached 7373 people. On February6, 2015, World Health Organization published data that Guinea, Liberia and Sierra Leone of the Ebola harder-hit area found cumulative suspected, possible or confirmed 22,525 cases of Ebola, 9004 people died.

As it is shown in Figure 1, we have got some Ebola virus data from March 2014 to December published by the World Health Organization (WHO) [3]. From the figure 1, we can see that the total number of Ebola infections increase slightly from March to July. However, the total number increases rapidly from July to December.

Faced with such huge numbers, the solution of Ebola should take immediate action. If there is no effective drug control measures and the epidemic will continue to spread it, and spread to the whole world. We build a model based on polymorphic ant colony algorithm to determine the optimal locations of delivery. By the experiment, we find our model can work well, they can help government forecast the epidemic spread, and can save a lot of resource.

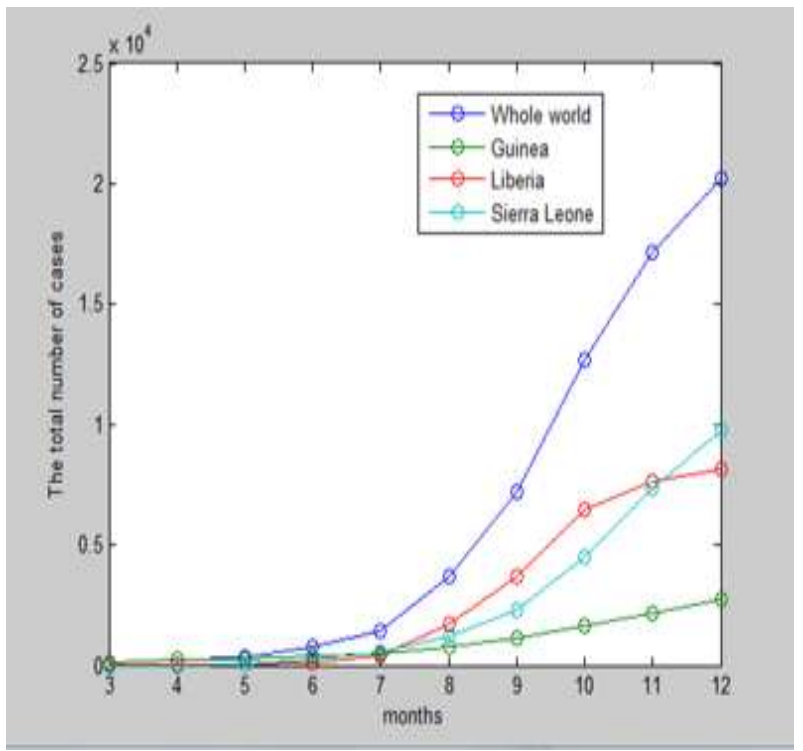

Figure 1. cumulative number of Ebola virus

We take the problem of determine the optimal locations of delivery medicines to shortest path problem. Shortest path problem is a typical combinatorial optimization problem. The ant colony algorithm has excellent properties of robustness, self-adaptation, parallelism, and positive feedback, it is suitable to solve some complex combinatorial optimization problem. In this paper, we adopt the polymorphic ant colony algorithm to solve the shortest path problem.

The rest of this paper is organized as follows: Section 2 lists some related work. Section 3 describes how to solve the shortest path problem of Ebola Virus prorogation using the polymorphic ant colony algorithm, and shows the experimental results in Section 4. Section 5 summarizes our algorithm and suggests our further work. 


\section{RELATED WORK}

\subsection{Polymorphic Ant Colony Algorithm}

Ants and other insects can achieve complex group behavior like coordination look for food and labor allocation by using pheromones to communicate each other [4]. These biological social behaviors have become an important source of ideas in robots and artificial intelligence. The ant colony algorithm (ACA) was first proposed by the Italian scholars Dorigo et al in nineties in the twentieth century [5]. Dorigo put forward the ant colony algorithm by simulating the seeking-food behavior of ants. Ant colony optimization (ACO) is a technique of problem solving inspired by the behavior of ants in finding paths from the nest to food and a new search metaphor for solving combinatorial optimization problems, and has been unexpectedly successful in recent years [6-10].

It is a heuristic algorithm inspired by the process of ants search for food and it is simulated evolutionary algorithm. The ant colony algorithm first successfully applied to the traveling salesman problem (TSP) [12]. Ant colony algorithm has the characteristics of distributed computing and easy to integrate with other simulated evolutionary algorithm, so it is suitable for solving NP-hard problem expressed by the available graph. The ant colony algorithm and it's various improved algorithms have been widely used and achieve better results to solve vehicle routing problem, fuzzy rule extraction, supply chain management and image recognition and other fields.

Polymorphic ant colony algorithm (PACA) is an improved ant colony algorithm [13] which solved the lack of polymorphism of the basic ant colony algorithm. It divided the ant colony society into three categories: scout ants, search ant and ergate. Scout ant's task is to detect each city as the center for local surveillance, and reconnaissance to detect the result has always been marked to search for scout ants to reach the next station when the city selected to provide supplementary information. Search ant's task is to do global search at every stop and to select the next according to scout pheromone and the pheromones of each export etc, until find and mark the best (shortest) route to the best route from the ergate feeding back to the nest. Ergate's task is feeding back to the nest according to the best route has been marked.

The PACA introduces a different type of ant colony, each ant colony has different regulatory mechanism of pheromone and combine the global search with local search, so the PACA is more in line with the actual information processing mechanism of ant colony and significantly improved the search, convergence speed, effectiveness and efficiency of the algorithm optimization. Simulation experiment results show that to get the same results of the case with the basic ant colony algorithm, the PACA required more less of iterations than the basic ant colony algorithm.

\subsection{Description of Shortest Path in Ebola Prorogation}

In this paper we consider the problem of shortest path in the uncertain environment. The problem can be described as follows: there are groups of cities allocate to ants to reach, each city only can be allocated to one ant and each ant reach one city at the same time. The goal of shortest path problem is to find the minimization of total cost in different allocation schemes.

For the convenience of modeling, we introduce the symbol:
$W: W$ means the set of cities to be allocated. We denote $W=\left\{T_{1}, T_{2}, \cdots, T_{n}\right\}$.

MAXPC : MAXPC means maximal selectable cities. It determined according to table 1, the data comes from paper [11]. We statistic the optimum solution in different size of cities, $C_{i}$ means each city in the best solution, make circle use $C_{i}$ as the center and $R$ as the radius. $R$ increase from zero until reach to the neighbor city of $C_{i}$, then record the number of cities in the circle and denote it as $P C_{i}, M A X P C$ is the maximum of $P C_{i}$.

Table 1 Statistic results of maxima1 selectable cities

\begin{tabular}{ccc}
\hline Number of cities & MAXPC & $\begin{array}{c}\text { Number of cities } \\
\left(P C_{i}=1\right)\end{array}$ \\
\hline 20 & 8 & 11 \\
100 & 9 & 55 \\
144 & 13 & 61 \\
1000 & 19 & 545 \\
\hline
\end{tabular}

We can see from table 1 that when the number of cities $n \leqslant$ $100, M A X P C<10$; when $100<n \leqslant 1000, M A X P C<20$, and it's probability is above $50 \%$, this means that it have a large probability of selecting the shortest edge which starting from the city $C_{i}$. We select neighbor city from the nearest $M A X P C$ cities not from all the remaining cities, by doing this, the solution space can decrease from $n$ ! to $n^{M A X P C}$, making the search speed significantly faster.

$c_{i j}: c_{i j}$ is defined as the total cost of Ant $t_{i}$ complete city $_{j}$. It contains time resource cost, resource consumption of various materials, communication costs, etc. the time cost is an important factor, so $c_{i j}$ can be computed as

$$
c_{i j}=\omega_{1} t_{i j}+\omega_{2} \lambda_{i j}
$$

where $t_{i j}$ is time resource cost, $\lambda_{i j}$ is other resource cost, $\omega_{1}$ and $\omega_{2}$ are weights which satisfy the condition $\omega_{1}+\omega_{2}=1$; $x_{i j}: x_{i j}$ is $0-1$ decision variable which defined as follows:

$$
x_{i j}=\left\{\begin{array}{l}
1, \text { if } \text { city }_{i} \text { done by } \text { Ant }_{j} \\
0, \text { otherwise }
\end{array}\right.
$$

$t a b u_{r}$ : We define $t a b u$ is the taboo table of each ant which memory the cities have already assigned. After each interaction, tabu will be cleared. $t a b u_{r}$ means the $t a b u$ of $A n t_{r}$.

The goal function is define as

$$
\min (F(x))=\sum_{k=1}^{n} \sum_{j=1}^{m} \sum_{i=i 1}^{i n_{i}} x_{i j} c_{i j}
$$

\section{SOLVE SHORTEST PATH PROBLEM BASED ON PACA}

The PACA-based shortest path problem mainly involves three types of Ant: Task allocation Ant, Task execution Ant and Environment Ant. Task allocation Ant is responsible for the task decomposition and task allocation; Task execution Ant is responsible for accept the task and execute it; Environment Ant is responsible for administrative tasks' and Ants' information, it does not involved in decision-making of task allocation in a distributed environment.

In the use of PACA for shortest path, the Task allocation Ant which assume the role of scout ants in PACA is a key player 
in decision-making of shortest path, and the Task execution Ant which assume the role of search ants in PACA is a key implementer in decision-making of shortest path, the Environment Ant does not participate in decision-making, so it assume the role of ergate in PACA.

We make the following assumptions:

(1)The cities to be allocated at the same probabilities;

(2)Every two cities has road, that cities are independent;

(3)Each Ant do the tasks according the rule of First Come First Served (FCFS).The specific method to apply the PACA to shortest path is: to the Task allocation Ant, put $m$ Task allocation Ants to $m$ positions, every Ant take it's position as the center to scout another $m-1$ positions, to constitute scout pheromone combine the scout result and prior knowledge, then take it as $s[i][j]$, and mark it on the link between city $_{i}$ and city $_{j} \cdot s[i][j](i, j=0,1,2, \cdots, m-1 ; i \neq j)$ is given by:

$$
s[i][j]=\left\{\begin{array}{l}
\frac{\tilde{d}_{i j}}{d_{i j}}, \text { if } \text { city }_{j} \text { in MAXPC of } \text { city }_{i} \\
0, \quad \text { otherwise }
\end{array}\right.
$$

where $\tilde{d}_{i j}$ is the shortest time to another $m-1$ cities in the center of city ${ }_{i} \cdot d_{i j}$ is the time of Ant from city $y_{i}$ to city $_{j}$. First, set the initial pheromone value on each path according to the result as follows:

$$
\tau_{i j(0)}= \begin{cases}C \square s[i][j], & \text { if } s[i][j] \neq 0 \\ \frac{C \square \tilde{d}_{i j}}{\hat{d}_{i j}}, & \text { otherwise }\end{cases}
$$

where $\hat{d}_{i j}$ is the longest time to another $m-1$ cities in the center of city $_{i} ; C$ is the initial pheromone value.

Then, the scout pheromone can provide aid to the Search Ant to calculate transition probability $p_{i j}^{k}$ and pheromone concentration on each path.

To the Task execution Ant, when Ant $k(k=1,2, \cdots, n)$ finish the current city at time $t, p_{i j}^{k}$ which means the probability of transfer city $_{i}$ to city $_{j}$ calculatedas follows:

$$
p_{i j}^{k}= \begin{cases}\frac{\left[\tau_{i j}(t)\right]^{\alpha}\left[\xi_{i j}(t)\right]^{\beta}}{\sum_{s \notin t a b u_{k}}\left[\tau_{i s}(t)\right]^{\alpha}\left[\xi_{i s}(t)\right]^{\beta}}, & \text { if } j \notin t a b u_{k} \\ 0, & \text { and } s[i][j] \neq 0 \\ & \text { otherwise }\end{cases}
$$

According to this formula, every time the Task execution Ant accept a city, only to search in a small range combined with scout pheromone and greatly reduce the search size. When all Ants complete a cycle, the pheromone on each path adjusted according to the following formula

$$
\tau_{i j}(t+1)= \begin{cases}\rho \square \varpi_{i j}(t)+(1-\rho) \square \Delta \tau_{i j}, & \text { if } s[i][j] \neq 0 \\ \rho \square t_{i j}(t), & \text { otherwise }\end{cases}
$$

where the $\Delta \tau_{i j}$ is the sum of pheromone value each Ant released at edge $(i, j)$ in this cycle. And $\Delta \tau_{i j}=\sum_{k=1}^{n} \Delta \tau_{i j}^{k} . \Delta \tau_{i j}^{k}$ is the pheromone value released by Ant $\mathrm{k}$ at path $(i, j)$ in this cycle and it given by the following formula

$$
\Delta \tau_{i j}^{k}=\left\{\begin{array}{c}
\frac{Q \square\left(\tilde{d}_{i j} / d_{i j}\right)}{L_{k}}, \\
\quad \text { if Agent } k \text { travles on } \\
0, \quad \text { otherwise }
\end{array}\right.
$$

where $Q$ is intensity of pheromone and affect the convergence speed at some extent; $L_{k}$ is the sum time used by Ant $\mathrm{k}$ in this cycle. Each Task execution Ant only to release suitable pheromone (combine local pheromone: $\tilde{d}_{i j} / d_{i j}$ and global pheromone: $L_{k}$ ) on the path which probably part of the optimal solution according to scout pheromone. According to formula (6), each Task execution Ant only to raise pheromone concentration on the path which probably part of the optimal solution (decide by whether $s[i][j]$ is $O$ ).

Therefore, the algorithm process can be described as follows:

1) Initialize $Q, C$ and the maximum evolution generation

2) Put $m$ Task allocation Ants on the $m$ cities' positions, every Ant take its position as the center to scout other $m-1$ positions, and calculate scout pheromone according to formula (4) and put the result into $s[i][j]$;

3) Set the initial pheromone on every path according formula (5);

4) Set evolution algebra initial value to 0 ;

5) Select each Task execution Ant's initial position randomly, and put the position into each Task execution Ant's table $t_{a b u}$;

6) Calculate the position each Task execution Ant will be transferred to and assume it as $j$ and the last position is $i$,put $j$ into each Task execution Ant's table $t_{a b u}$;

7) Calculate the objective function value $L_{k}(k=1,2, \cdots, n)$ of each Task execution Ant and record the current best solution;

8) If reach to the defined evolution generation or the solution has no significant improvement in last several generations, then jump to step 11). Otherwise jump to step 9);

9) Modify the pheromone concentration on each path according to formula (7);

10) Set $\Delta \tau_{i j}$ to 0 , and set table $t a b u_{k}$ empty, $N_{c} \leftarrow N_{c}+1$,and then jump to step 5);

11) Output the best solution.

\section{EXPERIMENTAL RESULTS}

\subsection{Performance comparison of PACA with basic ant colony algorithm}

In order to examine the performance of the polymorphic ant colony algorithm in this paper, we complete some contrast experiments. First we imitate the topology of the actual network and produce the required data: Number $n$ of the Task allocation Ants and Number $m$ of the Task execution Ants. The shortest path problem is solved by using basic ant colony algorithm and the polymorphic ant colony algorithm respectively. Then the obtained results are compared with the current results. By means of the adjustment, the parameters were set as follows: $n=3, m=5, Q=100, C=3, \alpha=1, \beta=3, \rho=0.3$, 
$M A X P C=10$. In experiment, each test iterates 100 times, and takes the average results of 10 experiments. Performance contrast of the experiment results of the two algorithms is shown in Table 2. The optimization result of each item is indicated with hold body.

Table 2 Experimental results

\begin{tabular}{ccccc}
\hline City numbers & \multicolumn{2}{c}{ Iteration numbers } & \multicolumn{2}{c}{ Times(s) } \\
\hline & $\begin{array}{c}\text { polymorphic } \\
\text { ant colony } \\
\text { algorithm }\end{array}$ & basic ant & polymorphic & basic ant \\
& 127.7 & 189.3 & 39.37 & 63.62 \\
algorithm & algorithm & algorithm \\
\hline 60 & 208.2 & 287.6 & 73.78 & 107.85 \\
95 & 261.5 & 396.8 & 98.03 & 121.17 \\
\hline
\end{tabular}

As can be seen from the table, in the same situation, the PACA spends much more time than the basic ant colony algorithm. The mainly reason is in PACA, every time when the search ant arrival a city, according to scout pheromone, it only search in a small range, which greatly reduces the search size.

\subsection{Comparison of the results with different parameters}

In order to examine how the parameters influence the experiment result, we complete the experiment at the case of group 1 has 25 cities, group 2 has 30 cities and group 3 has 40 cities. We use 3 Task allocation Ants and 5 Task execution Ants, takes the average results of 10 experiments. Table 3 shows the influence of the different parameter values upon the experimental results. As can be seen, the iteration number is smallest (209.1), when $\alpha=1, \beta=3, \rho=0.3, M A X P C=10$.

Table 3.The experimental results with different parameters of PACA

\begin{tabular}{ccccc}
\hline \multicolumn{3}{c}{ Parameter setup } & Iteration numbers \\
\cline { 1 - 3 }$\alpha$ & $\beta$ & $\rho$ & MAXPC & \\
\hline 1 & 2 & 0.3 & 11 & 315.8 \\
1 & 3 & 0.1 & 10 & 209.1 \\
2 & 1 & 0.2 & 9 & 383.8 \\
2 & 3 & 0.3 & 8 & 473.5 \\
3 & 2 & 0.1 & 7 & 598.3 \\
3 & 1 & 0.2 & 6 & 637.6 \\
\hline
\end{tabular}

\section{CONCLUSIONS}

In order to control the further spread of the Ebola virus, make the vaccine efficiently and quickly transport to the hardest hit by the disease, reduce constantly the number and scale of the Ebola epidemic, and ultimately control the spread of the virus, in this paper we considered the problem of shortest path to solve Ebola Virus prorogation problem. We introduced PACA to solve this problem, the experiment result shows that the algorithm are suitable for cases where Ants are motivated to act in order to maximize the benefits of the system as a whole. Comparative analysis the experiment results, we can see that the PACA reduce the weakness in search time and prone to stagnation in problem solving.

Using the three models that we proposed, the Governments and the WHO can accurately predict the development trend of the Ebola outbreak, choose the optimal delivery station and distribution plan of the vaccine, efficiently introduce disease control measures and, in turn, control the spread of the Ebola virus in order to achieve the purpose of the eradication of the Ebola virus.

\section{REFERENCES}

[1] Zhang L, Wang H. Forty years of the war against Ebola [J]. Journal of Zhejiang University Science B, 2014, 15(9): 761-765.

[2] Carroll M W, Matthews D A, Hiscox J A, et al. Temporal and spatial analysis of the 2014-2015 Ebola virus outbreak in West Africa[J]. Nature, 2015.

[3] Ebola virus disease. World Health Organization. September 2014.

[4] Han D C, Liu T H, Barber K S, A framework for problem solving activities in multi-Ant systems, The Laboratory for Intelligent Processes and Systems, The University of Texas at Austin,1999.

[5] M. Dorigo, V. Maniezzo, A. Colorni, The ant system: optimization by a colony of cooperating Ants, IEEE Transactions on Systems, Man and Cybernetics: Part B 26(1), pp.29-41,1996.

[6] M. Dorigo, T. Stu" tzle, Ant Colony Optimization, MIT Press, USA, July 2004.

[7] S.C. Zhan, J. Xu, J. Wu, The optimization selection on the parameters of the ant colony algorithm, Bulletin of Science and Technology, 19 (5) ,pp.381-386,2003.

[8] M. Dorigo, G.D. Caro, Ant colony optimization: a new meta-heuristic, in: Proceedings of the 1999 Congress on Evolutionary Computation, Washington, DC, IEEE Press, Piscataway, NJ, 1999, pp.1470-1477.

[9] M. Dorigo, M. Birattari, T. Stu“ tzle, Ant colony optimization, IEEE Computational Intelligence Magazine 1 (4),pp.28-39,2006.

[10] T. Stu" tzle, M. Dorigo, A short convergence proof for a class of ant colony optimization algorithms, IEEE Transctions on Evolutionary Computation 6 (4),pp.358365,2002 .

[11] Haluk Topcuoglu, Salim Hariri, and Min-You Wu. Performance-Effective and Low-Complexity Task Scheduling for Heterogeneous Computing. IEEE Transactions on Parallel and Distributed Systems, 13(3),pp.260-274,2002.

[12] Dorigo M, Gambardella L M.,Ant colony system:a cooperative learning approach to the traveling salesman problem, IEEE Transactions on Evolutionary Computation, vol.1(1),pp.53-66,1997.

[13] XU Jingming, CAO Xianbin, WANG Xufa, Polymorphic Ant Colony Algorithm, JOURNAL OF UNIVERSITY OF SCIENCE AND TECHNOLOGY OF CHINA, Vol.35(1), pp.,59-65, Feb,2005. 